\title{
Evolution of MRI changes and development of bilateral hippocampal sclerosis during long lasting generalised status epilepticus
}

\author{
B Pohlmann-Eden, A Gass, C N A Peters, R Wennberg, I Bluemcke
}

J Neurol Neurosurg Psychiatry 2004;75:898-900. doi: 10.1136/jnnp.2003.015354

\begin{abstract}
This report describes a previously healthy 28 year old patient with a 5 month period of intractable generalised status epilepticus (SE) of unknown aetiology with fatal outcome. Repeated magnetic resonance imaging (MRI) showed no preexisting abnormality, but did show progressive cortical and hippocampal atrophy and T2 hyperintensity in both hippocampal formations, suggestive of progressive tissue damage. Post-mortem histopathological analysis revealed substantial neuronal cell loss including CA1 and CA4 sectors of the hippocampus compatible with bilateral hippocampal sclerosis. There was no evidence of systemic complications including arterial hypotension and hypoxia, hypoglycaemia, hyperpyrexia, or other confounding factors to account for these findings. This case provides further evidence of SE induced hippocampal damage in humans.
\end{abstract}

$\mathrm{H}$ ippocampal sclerosis (HS) is the most common pathological finding in chronic temporal lobe epilepsy (TLE). Whether hippocampal atrophy and neuronal cell loss is the cause or consequence of repeated seizure activity has been a controversial debate for almost a century. Experimentally, Meldrum and coworkers ${ }^{1}$ first described selective neuronal damage in the hippocampus (HC) in a model of SE induced by bicculline, and showed that prolonged seizure activity alone could lead to excitotoxic cell death. Meanwhile, numerous animal studies using different SE models such as kainic acid, ${ }^{2}$ pilocarpine, ${ }^{3}$ or electrical stimulation $^{4}$ demonstrated that long lasting seizure activity resulted in selective neuronal loss in the $\mathrm{HC}$ with a similar distribution as in humans, mostly affecting CAl and CA3 regions of the HC (for overview see Lado et al, ${ }^{5}$ Blümcke et $a l^{6}$ ). According to new experimental data, even repeated brief seizures may induce neuronal loss and a pattern of hippocampal damage similar to that found in TLE. ${ }^{7}$

Thorough documentation of patients with SE induced brain damage is still rare. A recent magnetic resonance imaging (MRI) study in a small series of children provided some evidence that prolonged febrile seizures may lead to hippocampal atrophy, although the role of pre-existing traumatic, developmental, and genetic factors remained uncertain. ${ }^{8}$ In a retrospective, neuropathologically confirmed study of three cases with HS and SE lasting up to 3 days, where confounding factors could be ruled out, Fujikawa et al ${ }^{9}$ described widespread neuronal loss in the HC, amygdala, dorsomedial thalamus and cerebellar Purkinje cells, and the entorhinal and periamygdaloid cortex. A small number of clinically and radiologically well documented case studies using MRI changes over time have provided further evidence that HS may occur as a result of neuronal damage induced by seizures during SE. ${ }^{10-14}$ These studies describe an initial pattern of T2 signal increase and associated swelling in the $\mathrm{HC}$ without contrast enhancement, interpreted as focal periictal oedema. ${ }^{8}{ }^{10-17}$ Although progressive hippocampal atrophy on follow up MRI scans was only found in single cases, ${ }^{10-13}$ three patients had additional pathological proof of neuronal loss in the HC, amygdala, and claustrum. ${ }^{11}{ }^{12}{ }^{14}$ Some of these reported cases suggest that HS can develop within 2 months of SE and may further progress during the following 3 to 4 years. ${ }^{11}{ }^{13}$ Even repeated single brief generalised tonicclonic seizures were reported to lead to HS according to MRI criteria. $^{18}$

\section{CASE REPORT}

We report a case of a 28 year old man with no previous neurological or medical history, no contact with infectious diseases, and no history of drug or alcohol withdrawal. He was a product of a normal delivery. The family history for epilepsy was negative. There was no evidence of delay in psychomotor development, previous head trauma, encephalitis, febrile convulsions, or other predisposing conditions. He presented to the emergency room with a first generalised tonic-clonic seizure (GTCS), followed by a series of GTCS on day 1 , developing into a drug resistant generalised SE on day 2 that prompted treatment with general anaesthesia. The initial post-ictal clinical examination revealed no focal signs. Long term continuous EEG monitoring, immediately started with the diagnosis of SE, repeatedly showed generalised seizure activity consisting of asynchronous irregular 4-5/s spike wave and sharp wave transients with shifting predominance without clear focal onset. Medical treatment included the entire range of established standard antiepileptic strategies (benzodiazepines, phenytoin, valproate, all barbiturates, lidocaine, propofol, ketamine chloride, clomethiazol, and oral lamotrigine, carbamazepine, topiramate, all at high doses, as single treatment or as combination therapy) as well as other therapeutic strategies such as high dose corticosteroids and even intermittent electroconvulsive therapy in the final stage of the SE. The SE could be only controlled by deep GA paralleled by classical burst suppression EEG. At no point did the patient develop significant hyperpyrexia, arterial hypotension, hypoxaemia, or hypoglycaemia. The patient died 5 months later most likely due to ICU complications including pancytopenia, with no evidence of the symptomatic aetiology underlying his SE having been discovered.

Extensive serum analyses including thorough screening for toxic agents such as domoic acid and repeated CSF samples were unrevealing. Autoimmunological parameters such as anti-nuclear antibodies, anti-neutrophil cytoplasmic antibody,

Abbreviations: GTCS, generalised tonic-clonic seizure; HC, hippocampus; HS, hippocampal sclerosis; MRI, magnetic resonance imaging; SE, status epilepticus; TLE, temporal lobe epilepsy 
and myelin antibodies were negative, as were all antibodies and complement binding reactions against tuberculosis, Borrelia, Toxoplasma, Candida and Actinomyces, human immunodeficiency virus, herpes simplex virus, cytomegalovirus, Epstein-Barr virus, varicella zoster virus, polio, rabies, and rubella. Initial CSF cell count increased from 9 cells $/ \mathrm{mm}^{3}$ to 37 cells $/ \mathrm{mm}^{3}$ during three follow up investigations.

MRI scans (Proton Density, FLAIR, $\mathrm{T}_{1}$ and $\mathrm{T}_{2}$ weighted (+ contrast)) were performed four times during the hospital stay. Initial MRI on the day of admission was normal. Repeat MRIs after 4,10 , and 16 weeks demonstrated the development of generalised atrophy (fig $1 \mathrm{~A}-\mathrm{C}$ ) and $\mathrm{T} 2$ hyperintense signal changes after 4 weeks in the hippocampal formations (fig ID).

Neuropathological investigation demonstrated widespread neuronal cell loss and astrogliosis in neocortex, basal ganglia, cerebellum, and especially in the hippocampi bilaterally, most pronounced in the CAl and CA4 regions (fig 2). In contrast, dentate gyrus granule cells and CA2 pyramidal cells were not affected. Hippocampal pathology was reminiscent of an early stage of Ammon's horn sclerosis in patients with intractable temporal lobe epilepsy. Thorough examination of all brain structures revealed no specific findings in regard to the presumed symptomatic aetiology of the SE such as encephalitis, traumatic scar formation, malformations, or neoplasia.

\section{DISCUSSION}

The hippocampal subfields CAl (Sommer's), CA3, and CA4 appear particularly susceptible to seizure induced damage, which is presumed to result from excessive presynaptic release of excitatory neurotransmitters, specifically glutamate and to a lesser extent aspartate. ${ }^{19}$ Abundant glutamate binding sites in rat $\mathrm{CAl}$ and $\mathrm{CA} 3$ regions support this concept of glutamate mediated excitotoxicity. ${ }^{20}$ In the kainic acid animal model of SE (kainic acid is an excitotoxic analogue of glutamate), a time dependency and threshold phenomenon of neuronal damage has been demonstrated. Neuronal cell loss occurred as early as 30 minutes after SE

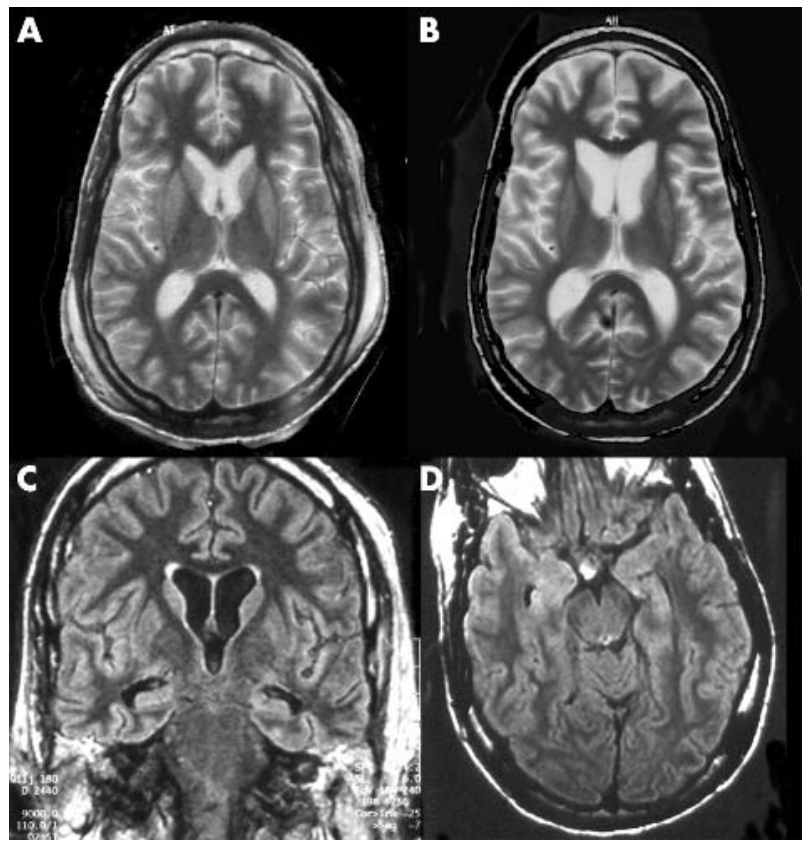

Figure 1 Demonstration of the development of generalised, predominantly supratentorial, cortical atrophy, on T2 weighted MRI over 3 months. (A) Week 4, (B) week 16. FLAIR images at week 10 show evidence of flattened small hippocampus in coronal plane (C) and hyperintensity in the head of the hippocampus in the transverse plane (D).
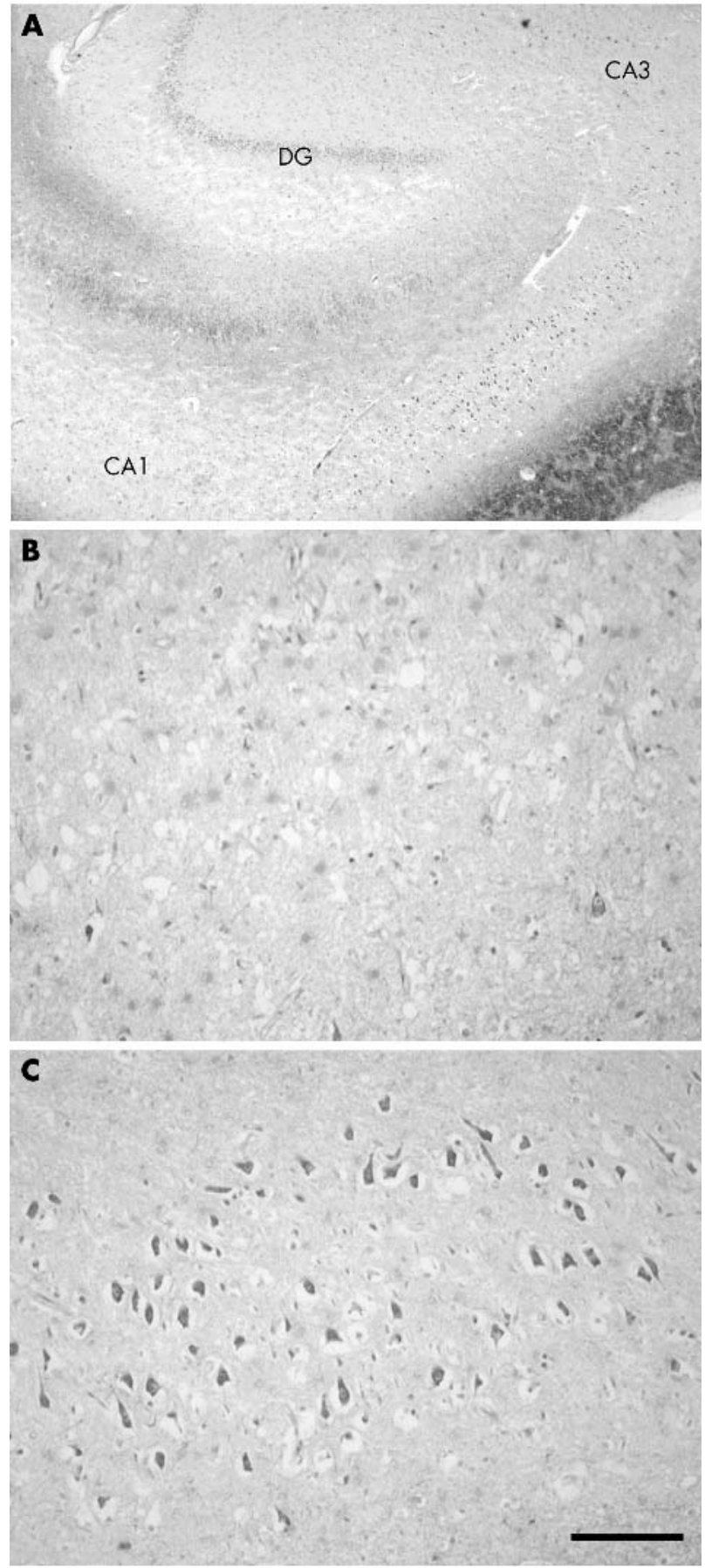

Figure 2 Left hippocampus (Nissl staining): note the extensive neuronal cell loss in CA4 (A) and CA1 (B), whereas granule cells and CA2 pyramidal cells (C) are preserved. Scale bar $=150 \mu \mathrm{m}$.

and showed its greatest topographical distribution in long lasting SE. ${ }^{21}$ Experimental imaging studies showed that intrahippocampal injection of low doses of kainate in mice reproduced the MRI pattern of HS with T2 signal increase, interpreted to result from both the excitotoxic effect of kainate and the ongoing seizure activity. ${ }^{22}$ A recent lithiumpilocarpine model of SE demonstrated as the earliest MRI change, after 2 hours, a blood-brain barrier breakdown in the thalamus, followed by oedema in the amygdala and the piriform and entorhinal cortices after 24 hrs. Histopathologically confirmed hippocampal cell loss correlated significantly with the evolution of the T2 signal increase. ${ }^{23}$ 
In humans, the most frequently observed MRI change in the early stage of SE is either asymmetrical or bilateral T2 signal increase in the $\mathrm{HC}^{, 10-17}$ sometimes found after as little as 24 hours, ${ }^{10}$ often encompassing the entire length of the HC and interpreted as focal transient cytotoxic and vasogenic oedema. High resolution MRI demonstrated involvement of the CA subfields within the HC in one case. ${ }^{12}$ Documentation of an associated early swelling of the affected HC was shown using different methods of hippocampal volumetry, ${ }^{8}{ }^{13} 16$ and by signal increase on diffusion weighted imaging together with focally reduced apparent diffusion coefficients. ${ }^{16}$ T2 signal increase seems to disappear within a few days after short term $\mathrm{SE},{ }^{16}$ but may persist in the HC for several weeks and spread to other structures such as the fornices in long term refractory SE. ${ }^{12}$ Development of hippocampal atrophy proven by MRI in a previously unremarkable HC was reported in both children ${ }^{10}$ and adults ${ }^{11}{ }^{13}$ following long lasting seizure activity.

Not surprisingly, there is a lack of MRI data in the chronic stage of ongoing SE in both animals and humans. As the first follow up MRI on our patient was performed almost 4 weeks after the first normal MRI, it is likely that the initial T2 increase and swelling of the HC had already disappeared and the excitotoxicity related neuronal damage had already begun to occur by this time. At this time point, we found signal increases without swelling in both hippocampal formations, at a maximum in the vicinity of the head of the HC. Serial coronal Tl weighted and FLAIR images showed significant volume loss in both hippocampi between week 4 and 10, most likely representing neuronal loss and astrogliosis as the correlate of the later histologically proven HS. The selective vulnerability of the hippocampal formations is compatible with the outlined concept of excitotoxicity with a critically lowered threshold for glutamate toxicity in this stage of ongoing and widespread seizure activity. However, this topographical pattern is unspecific and shares certain characteristics with observations in post-hypoxic damage, ${ }^{24}$ such that a mechanism of repeated hypoxic injury due to ictal overactivation and metabolic compromise in a vulnerable tissue compartment could also be considered.

In summation, both the neuropathological findings and corresponding MRI features in our case further support the hypothesis that neuronal cell loss in HS may occur as the result of prolonged severe seizure activity.

\section{Authors' affiliations \\ B Pohlmann-Eden, A Gass, C N A Peters, Department of Neurology, Mannheim Hospital, University of Heidelberg, Germany \\ B Pohlmann-Eden, R Wennberg, Krembil Neuroscience Centre, Toronto Western Hospital, University of Toronto, Canada I Bluemcke, Department of Neuropathology, University of Erlangen, Germany \\ Competing interests: none declared}

Correspondence to: Dr B Pohlmann-Eden, Toronto Western Hospital, University of Toronto, 5W-443, 399 Bathurst St, Toronto, ON M5T 2S8, Canada; pohleden@gmx.net
Received 25 March 2003

In revised form 20 October 2003

Accepted 20 October 2003

\section{REFERENCES}

1 Meldrum BS, Vigoroux RA, Brierley JB. Systemic factors and epileptic brain damage. Prolonged seizures in paralysed, artificially ventilated baboons. Arch Neurol 1973;29:82-8.

2 Ben-Ari Y. Limbic seizure and brain damage produced by kainic acid: mechanisms and relevance to human temporal lobe epilepsy. Neurosci $1985 ; 14: 375-403$.

3 Fujikawa DG. The temporal evolution of neuronal damage from pilocarpineinduced status epilepticus. Brain Res 1996;725:11-22.

4 Lothman EW, Bertram EH, Bekenstein JW, et al. Self-sustaining limbic status epilepticus induced by "continuous" hippocampal stimulation: electrographic and behavioural characteristics. Epilepsy Res 1989;3:107-19.

5 Lado FA, Laureta EC, Moshe SL. Seizure-induced hippocampal damage in the mature and immature brain. Epilept Disord 2002;4:83-97.

6 Blümcke I, Thom M, Wiestler OD. Ammon's horn sclerosis: a maldevelopmental disorder associated with temporal lobe epilepsy. Brain Pathol 2002;12:199-211.

7 Sutula TP, Pitkanen A. More evidence for seizure-induced neuronal loss. Neurology 2001;57:169-70.

8 VanLandingham KE, Heinz RE, Cazavos JE, et al. Magnetic resonance imaging evidence of hippocampal injury after prolonged focal febrile convulsions. Ann Neurol 1998;43:413-26.

9 Fujikawa DG, Tabashi HH, Wu A, et al. Status epilepticus-induced neuronal loss in humans without systematic complications or epilepsy. Epilepsia 2000;41:981-91.

10 Nohria V, Lee N, Tien RD, et al. Magnetic resonance imaging evidence of hippocampal sclerosis in progression: a case report. Epilepsia 1994;35:1332-6.

11 Tien RD, Felsberg GJ. The hippocampus in status epilepticus: demonstration of signal intensity and morphological changes with sequential fast spin-echo MR imaging. Radiology 1995; 194:249-56.

12 Stafstrom CE, Tien RD, Montine TJ, et al. Refractory status epilepticus with progressive magnetic resonance imaging signal change and hippocampal neuronal loss. J Epilepsy 1996;9:253-8.

13 Wieshmann UC, Woermann FG, Lemieux L, et al. Development of hippocampal atrophy: a serial magnetic resonance imaging study in a patient who developed epilepsy after generalized status epilepticus. Epilepsia 1997;38:1238-41.

14 Nixon J, Bateman D, Moss T. An MRI and neuropathological study of a case of fatal status epilepticus. Seizure 2001;10:588-91.

15 Kim JA, Chung Jl, Yoon PH, et al. Transient MR signal changes in patients with generalized tonicoclonic seizure or status epilepticus: periictal diffusionweighted imaging. Am J Neuroradiol 2001;22:1149-60.

16 Scott RC, Gadian DG, King MD, et al. Magnetic resonance imaging findings within 5 days of status epilepticus in childhood. Brain 2002;125:1951-9.

17 Perez ER, Maeder P, Villemure KM, et al. Acquired hippocampal damage after temporal lobe seizures in 2 infants. Ann Neurol 2000;48:384-7.

18 Briellmann RS, Newton MR, Wellard RM, et al. Hippocampal sclerosis following brief generalized seizures in adulthood. Neurology 2001;57:315-17.

19 Sloviter RS. "Epileptic" brain damage in rats induced by sustained electrical stimulation of the perforant path. I. Acute electrophysiological and light microscopic studies. Brain Res Bull 1983;10:675-97.

20 Lallement G, Carpentier P, Collet A, et al. Effects of soman-induced seizures on different extracellular amino acids levels and on glutamate uptake in rat hippocampus. Brain Res 1991;563:234-40.

21 Ingvar M, Morgan PF, Auer RN. The nature and timing of excitototxic neuronal necrosis in the cerebral cortex, hippocampus, and thalamus due to fluorothyl-induced status epilepticus. Acta Neuropathol 1988;75:362-9.

22 Bouilleret V, Nehlig A, Marescaux, et al. Magnetic resonance imaging followup of progressive hippocampal changes in a mouse model of mesial temporal lobe epilepsy. Epilepsia 2000;41:642-50.

23 Roch C, Leroy C, Nehlig A, et al. Magnetic resonance imaging in the study of the lithium-pilocarpine model of temporal lobe epilepsy in adult rats. Epilepsia 2002;43:325-35.

$24 \mathrm{Ng} \mathrm{T}$, Graham DI, Adams JH, et al. Changes in the hippocampus and the cerebellum resulting from hypoxic insults: frequency and distribution. Acta Neuropathol 1989;78:438-43. 


\section{PostScript}

\section{CORRESPONDENCE}

\section{Validity of language lateralisation by unilateral intracarotid Wada test}

The case report by Loddenkemper et $a l^{1}$ is most important as it poses the question of whether unilateral Wada testing is sufficient for language lateralisation. It is certainly possible that similar complications resulting from epilepsy surgery based on Wada tests are underreported. The implications of erroneous language lateralisation by unilateral amobarbital injection, which is increasingly used in several epilepsy centres including our own, would be far reaching.

There are, however, some caveats regarding the interpretation of the Wada test results reported. The first two refer to the assessment of cortical inactivation in this patient with pre-existing damage to the motor cortex; the third refers to the extent of brain areas inactivated by the intracarotid Wada procedure.

(1) Little is known about the relative susceptibility to the effect of amobarbital of language areas compared with damaged motor cortex. Thus it may be that a low dose of amytal (which $100 \mathrm{mg}$ is) does impair a partially damaged motor cortex when language areas still maintain sufficient function to solve the tasks tested during the Wada procedure. In our experience, higher doses ( 140 to $200 \mathrm{mg}$ ) are often needed in adults to induce a complete contralateral hemiplegia. In order to avoid a possible risk of incomplete inactivation of the middle cerebral artery (MCA) territory and of language areas in particular, it may be advantageous to choose higher injection dosages.

(2) There is a striking discrepancy between well preserved proximal arm power (4-/5) after hemispherectomy as compared with complete proximal hemiplegia $(0 / 5)$ reported during the Wada procedure which requires additional explanation. This may indicate that the lack of voluntary movement observed during the Wada procedure does not result from a complete inactivation of the contralateral motor cortex but may be related to contralateral motor neglect.

(3) With regard to clinical history and magnetic resonance imaging, there is some indication that this patient may have had reorganisation of the language areas following the initial brain injury at the age of five years. Whereas the Wada test was interpreted as providing evidence for an interhemispheric shift to the right hemisphere, an intrahemispheric transfer of expressive functions to posterior temporal areas may have taken place. As the vascular supply of this region often is not restricted to MCA branches, incomplete inactivation of this area may occur. $^{2}$ The results of the procedure could thus be explained by the incomplete inactivation of posterior left hemispheric language areas. It would be interesting to know if the authors coinjected a SPECT tracer $^{3}$ in order to define the territory perfused by amobarbital.

\section{A Schulze-Bonhage, A Quiske Epilepsy Centre, University Clinic of Freiburg, Germany}

Correspondence to: Dr Andreas Schulze-Bonhage; schulzeb@nz.ukl.uni-freiburg.de

\section{References}

1 Loddenkemper T, Dinner DS, Kubu C, et al. Aphasia after hemispherectomy in an adult with early onset epilepsy and hemiplegia. J Neurol Neurosurg Psychiatry 2004;75:149-51.

2 Hart J, Lesser RP, Fisher RS, et al. Dominant-side intracarotid amobarbital spares comprehension of word meaning. Arch Neurol 1991;48:55-8

3 Biersack HJ, Linke D, Brassel F, et al. Technetium$99 \mathrm{~m}$ HM-PAO brain SPECT in epileptic patients before and during unilateral hemispheric anesthesia (Wada test): report of three cases. J Nucl Med 1987;28:1763-7.

\section{Authors' reply}

We appreciate the above comments and are delighted that our case has sparked a discussion about the validity of the Wada test. The intention of our report was to question the use of the (unilateral) intracarotid amobarbital test (IAT) in selected cases, and we are pleased that Dr Schulze-Bonhage and Dr Quiske recognise the potential importance of this case. We would like to address their comments in detail.

Comment 1: No clinical data are available on the effect of amobarbital on the damaged motor cortex. Thus the effectiveness of an initial high dose of amobarbital, as well as incomplete inactivation of different MCA areas, remains an interesting speculation. Although we do not know whether higher amobarbital doses are more effective in cases like ours, we know that higher doses may increase the probability of drug related side effects such as encephalopathy and impaired consciousness, particularly in patients with pre-existing left hemispheric lesions. Additionally, amobarbital may have a stronger effect in older patients, ${ }^{2}$ supporting the use of a lower initial dose in our 55 year old patient. Furthermore, the recent replacement of amobarbital by methohexital, owing to a shortage of amobarbital in many centres, may subject patients to a dose dependent increase in seizure frequency during Wada testing when there is a high initial dose of methohexital. ${ }^{3}$ Clinical examination of the patient remains the most important factor in the careful titration of the amobarbital dose, in order to prevent adverse effects during its administration.

Comment 2: We thank the authors for highlighting the potentially interesting finding of the acute worsening of unilateral motor function during the Wada test. As documented, we would like to point out that the proximal strength of 4-/5 was only achieved after eight months, following vigorous rehabilitation. The discrepancy from the results during the Wada test arises because Dr Schulze-Bonhage and Dr Quiske are comparing the examination during the acute amobarbital inactivation with chronic findings eight months after recovery from hemispherectomy. The patient was completely hemiplegic immediately after hemispherectomy and then gradually regained the reported outcome strength of $4-/ 5$. Thus the amobarbital test mimicked the immediate postoperative outcome well. Proximal weakness has been reported during IATs, ${ }^{4}$ and the documented gradual recovery of strength over 145 seconds is unlikely in patients with motor neglect. ${ }^{5}$

Comment 3: IATs are often undertaken in patients undergoing epilepsy surgery. However, ipsilateral language areas are only rarely resected, especially if the test is positive. Even if the test is negative, the resected area does not necessarily include typical language areas. Therefore, little information on the validity of the IAT is available. IAT validation studies in predicting aphasia may be divided into four categories: true negatives, true positives, false negatives, and false positives. A false negative is someone who passed the IAT but nonetheless became aphasic. The incongruity of the clinically inactivated motor area and the target area (language) is a potential problem within the routine Wada test itself, which may be highlighted by cases like ours, and which is possibly exacerbated by an underlying subcortical lesion. Intrahemispheric reorganisation or interhemispheric language reorganisation is a possible explanation for atypical language representation in our case. The possibility of residual function and intrahemispheric reorganisation in our case is supported by intact visual fields before hemispherectomy, as previously discussed. ${ }^{5}$

Another issue related to the validity of the IAT is the distribution of the amobarbital. Conventional angiography, digital subtraction angiography (DSA), and SPECT have been compared in terms of perfusion patterns during IAT. With regard to posterior cerebral artery (PCA) filling in 46 patients, SPECT showed filling in $22 \%$, DSA showed filling in $37 \%$, and conventional angiography showed contrast in $50 \%$ of patients. ${ }^{6}$ Based on these data, a conventional angiography before the IAT (as carried out and reported in our case) can rule out perfusion of the PCA. Based on this study, no HMPAO distribution would have been observed in the PCA territory. SPECT is therefore unnecessary.

To our knowledge, we have reported the first case in which a patient continued to speak during the Wada test and then became aphasic after ipsilateral hemispherectomy. Aphasia after hemispherectomy was not anticipated. We agree with Drs SchulzeBonhage and Quiske that it is possible that similar complications resulting from epilepsy surgery based on Wada tests are underreported. The teaching point of our case report is that a negative unilateral routine Wada test result for language may be misleading in an older patient with a predominantly subcortical lesion that was acquired after the primary development of language function. We encourage other epilepsy centres to reflect on their current practice of unilateral Wada testing for presurgical language lateralisation in selected cases and possibly replace or supplement the Wada test by additional language lateralisation techniques. 
We thank Dr Schulze-Bonhage and Dr Quiske for their comments.

T Loddenkemper, D S Dinner, E Wyllie Department of Neurology, The Cleveland Clinic Foundation, Cleveland, Ohio, USA

T Loddenkemper Department of Pediatrics, The Cleveland Clinic Foundation

Correspondence to: Dr T Loddenkemper loddent@ccf.org

\section{References}

1 Lee GP, Loring DW, Meador KJ, et al. Severe behavioral complications following intracarotid sodium amobarbital injection: implications for hemispheric asymmetry of emotion. Neurology 1988;38:1233-6.

2 Segal JB, Moo LR, Hart J. The effect of age on rate of functional recovery after intracarotid amobarbital injection. Epilepsia 2002;43:659-61

3 Loddenkemper T, Morris H, Moddel G. Seizures during Wada testing. Epilepsia, 2003;44/suppl 9): 295

4 DeToledo JC, Dow R. Sternomastoid function during hemispheric suppression by amytal: insights into the inputs to the spinal accessory nerve nucleus. Mov Disord 1998;13:809-12

5 Loddenkemper T, Dinner DS, Kubu C, et al. Aphasia after hemispherectomy in an adult with early onset epilepsy and hemiplegia. I Neurol Neurosurg Psychiatry 2003;75:149-51.

6 Jeffery PJ, Monsein LH, Szabo Z, et al. Mapping the distribution of amobarbital sodium in the intracarotid Wada test by use of Tc-99m HMPAO with SPECT. Radiology

$1991 ; 178: 847-50$

\section{Tangier disease}

With reference to the article entitled "Tangier disease-a diagnostic challenge in countries endemic for leprosy", ', I should like to point out that the name "Tangier disease" originates from a small island in Chesapeake Bay, USA and not from Morocco as stated by the authors.

The first case of this uncommon disease with neurological involvement was reported by Kocen et al in the Lancet in $1967^{2}$ and by Engel $^{3}$ from the USA soon after. Detailed neuropathology on this condition in our and one other case was reported by Kocen et al in $1973 .^{4}$

R S Kocen

127 Willifield Way, London NW11 6XY, UK rskocen@aol.com

Competing interests: none declared

\section{References}

1 Sinha S, Mahadevan A, Lokesh L. Tangier disease - a diagnostic challenge in countries endemic for leprosy. J Neurol Neurosurg Psychiatry

$$
\text { 2004;75:301-4 }
$$

2 Kocen RS, Lloyd JK, Lascelles PT, et al. Familial lipoprotein deficiency (Tangier disease) with neurological abnormalities. Lancet 1967:1341-5.

3 Engel WK, Dorman JD, Levy RI, Fredrickson DS. Neuropathy in Tangier disease. Alpha-lipoprotein deficiency manifesting as familial recurrent neuropathy and intestinal lipid storage. Arch Neurol Jul, 1967;17:1-9.

4 Kocen RS, King RHM, Thomas PK, et al. Nerve biopsy findings in two cases of Tangier disease. Acta Neuropathol (Berl) 1973;26:317-27.

\section{BOOK REVIEWS}

\section{Neurology for non-neurologists, 4th edition}

Wigbert C Wiederholt. Published by W B Saunders, Philadelphia, 2000, pp 323, £32.99. ISBN 0-7216-8874-8

This book is targeted at "students, residents and practicing physicians whose primary interest is not neurology", although at 342 pages, no colour, and very few diagrams or pictures, I felt this may be a little off putting to non-neurologists and neurologists alike! However, this is an extensive review that considers basic science, pathophysiology, and management issues, which is a useful reference tool. The first three chapters (60 pages) are an introduction to neurology and include a chapter on neuroanatomy, as well as a thorough discussion about the neurological history, examination, and common investigations. There then follows chapters on specific areas such as headache, cerebrovascular disease, dementias, and demyelinating diseases. The chapters are detailed but again may be considered overwhelming for the general physician, for example in the headache chapter four pages are dedicated to the complete International Headache Society's classification of headache.

Interestingly, throughout the book there are very few references to when a patient should be referred on or would benefit from the input of a neurologist. Considering this book deals with rare, serious, and genetic neurological disorders, I find this approach rather disappointing. It is also written predominantly for the American market, another factor that perhaps makes the lack of reference to the need for specialised neurological involvement surprising. Drug names, dosage schedules, and units are in the accepted US format.

In summary, this is a concise but detailed reference book covering a wide range of neurological conditions. It is a useful tool for students and junior doctors but it perhaps encourages the reader to manage complex and rare conditions themselves and neglects the need for specialist input.

V L Stevenson

\section{Neuropsychopharmacology}

W W Fleischhacker, D J Brooks, eds. Wien and New York: Springer Verlag 2003, pp 114, $€ 89$. ISBN 3-21 1-83903-8

The European Institute of Healthcare devoted its 5 th Neuropsychiatry Symposium to neuropsychopharmacology. Topics were chosen by the organisers and editors, Professors Fleischhacker and Brooks, to bridge the gap between basic neuroscience and the clinical fields of neurology, but mostly psychiatry. The slim volume comprises seven chapters that cover a wide range of topics from histamine receptors to developments in substance dependence research and the pharmacotherapy of schizophrenia. The chapters are uniformly up to date and written in a challenging and interesting way. Some of the chapters are extremely compendious, for example that on migraine contains 199 references.
Various advances in the therapeutics of neuropsychiatric conditions are reviewed. As a psychiatrist/psychopharmacologist, I must admit to finding the three last chapters the most fascinating. Professor Deakin reviews serotonin function in relation to antisocial personality disorder and depression. He adduces interesting arguments that serotonin abnormalities are related to both conditions, the difference being an anatomical selection in the dorsal raphe nucleus. This hypothesis gives rise directly to therapeutic suggestions for treating depression. These are of course largely in place. However, the treatment of antisocial personality disorder opens up Orwellian prospects and will need carefully monitoring.

It was particularly interesting to see a chapter devoted to future directions and substance misuse from Professor Nutt's group in Bristol. My only criticism of this chapter was that it was too short and only whetted one's appetite for further information concerning receptors, brain pathways, and promising avenues of drug development.

The final chapter by one of the editors deals with new developments in the pharmacotherapy of schizophrenia. The immense clinical experience of Professor Fleischhacker is shown by his acknowledgement that improved formulations, such as rapidly dissolving tablets and syrups, have an important part to play, in view of the poor adherence to treatment of patients with schozphrenia. He also reviews polypharmacy that is the norm in many practices and points the way to further developments with new classes of compounds.

Some curmudgeonly remark the English is often not carefully sub-edited and there are numerous typos (for example, see page 65). Some of the articles contain very long paragraphs that make the content difficult to follow. There is also no uniformity of reference style. One would expect SpringerVerlag to be able to afford the services of an experienced native English speaking subeditor.

The very last page states that the paper in the book was acid free in conformance with international standards for paper permanency. I suspect that the paper itself will outlive the content of these chapters, but that is no bad thing.

Overall, the book provides a rapid updating of knowledge across the field in neuropsychiatry. Inevitably, it will be out of date fairly quickly, but in the meantime it is important reading even for the cognoscenti.

M Lader

\section{The psychology of bulimia nervosa: a cognitive perspective}

Myra Cooper. Published by Oxford University Press, Oxford, 2003, pp 332, £29.50. ISBN 019-263265-5

The first description of bulimia nervosa was published in 1979 by Russell. Since that time there has been a burgeoning literature on all aspects of the disorder. This has coincided with the growth of cognitive psychology and cognitive behaviour therapy (CBT). As might be expected, the cognitive approach has been used in the investigation of bulimia nervosa with considerable success, and CBT has become the "gold standard" for adults requiring treatment on the basis of many 
randomised controlled trials. This book is a timely review of the literature, at a time when it is still just possible for a single author to achieve adequate understanding of the field.

The background chapters cover the diagnostic features, epidemiology, and theories of aetiology of the disorder. There is description of the main treatments, with special emphasis on CBT. Fairburn's established model of CBT balances the focus on changing eating patterns with cognitions and is fairly described. This is the foundation for Cooper's own original work, which places more emphasis on core beliefs, for example regarding the self and how they relate to beliefs about eating and dieting. Although Cooper's model has some evidence in support of its rationale, there is no evidence from treatment trials that it achieves greater benefits than Fairburn's approach, and so CBT practitioners might wonder why they should change to the new approach.

Overall this is a well written and well organised book. Each chapter has numerous useful summary tables of the main points covered in the text. Weaknesses of the book are its rather poor coverage of developmental issues, and negative bias with regard to drug treatments. Despite these points, the book achieves very well its main aim of providing a cognitive perspective. The book would be very useful for trainees and more experienced clinicians, and should have a place in any well stocked library.

$M$ Hodes

\section{The clinical practice of critical care neurology}

Eelco F M Wiidicks. Published by Oxford University Press, Oxford, 2003, pp 579, $£ 95.00$ (hardback, 2nd edn). ISBN 0-19515729-X

It was once said of the English cricket team "they played well but the only trouble is that they can't bat, can't bowl and can't field". In the United Kingdom we make similar comments about neurological intensive care-it's a good idea and important but we have no units, no specialists, and no training. This is, of course, an exaggeration but we continue to fall further behind the United States and Europe in the provision of care for patients acutely ill with neurological disorders. It must be shown that these units make a difference to the outcome of patients but it is both intuitively obvious and reflected in evidence-based medicine that providing the best environment for care will lead to better outcome. Wijdicks, together with others, has defined the field of neurological critical care with his textbooks and original contributions. The clinical practice of critical care neurology is a landmark in the field and the rapid publication of an enlarged and improved second edition reflects the importance of this book. Wijdicks writes from an extensive experience of managing patients with critical neurological illness. The book has all the advantages of a single author text, being coherent and logical with a thorough coverage of the subject. The layout of the second edition is a considerable improvement-the text is easier to follow and many of the practical procedures are more clearly described and illustrated. The book is divided into six sections, three deal with general aspects of neurological intensive care, discussing the practical details of setting up and running a neurological intensive care unit; the equipment and technology used in monitoring and treating the patients; and the clinical and ethical issues at the end of life. The major part of the book deals with the management of specific disorders, post-operative complications, and the management of the systemic complications seen in these patients. It is inevitable that there will be individual quibbles in such a wide ranging review of neurological management but the majority of these reflect different "fashions" between the United States and the United Kingdom rather than any fundamental differences in the interpretation of the evidence base.

Neurological intensive care is a subspecialty where the clinician has to cover a broad spectrum of the specialty and this is superbly reflected in the second edition of Wijdicks' textbook. A single author monograph of this sort cannot hope to be exhaustive but Wijdicks has achieved a remarkable job of ensuring detail is not compromised despite the breadth of coverage. This book provides essential reading and reference for all neurologists and intensivists charged with the management of patients on intensive treatment units with primary neurological disorders.

R Howard

\section{Stroke genetics}

Edited by Hugh Markus. Published by Oxford University Press, Oxford, 2003, pp 342, $£ 79.50$ (hardback). ISBN 0-19-851586-3

This is an extremely useful book that goes well beyond its stated aim as an introduction to stroke genetics for both the interested clinician seeing stroke patients, and the basic scientist entering this field of research.

The book comprises 14 chapters with contributions from 18 authors. The basic science areas are well covered, including the genetic epidemiology of stroke as well as monogenic and polygenic contributions to ischaemic stroke. What marks the book out from other "neurogenetic" texts, however, is the success with which it takes genetics out of the laboratory and into the general neurology clinic. This is exemplified by the last chapter, which presents a practical approach to investigating a patient presenting with stroke for underlying genetic disorders. The three chapters dealing with the genetics of the various causes of intracerebral haemorrhage, including approaches to screening for familial intracranial aneurysms, are of particular clinical relevance, as is the excellent introduction to genetic counselling.

Cerebral venous thrombosis as a cause of stroke was mentioned in several chapters, but unfortunately not covered in a chapter of its own. I would have welcomed more detailed guidance on the utility of screening for genetically determined prothrombotic disorders, and how to deal with the uncertain predictive values of test results. However, this is a minor criticism of an otherwise successful book that should be of interest to general neurologists as much as stroke geneticists.

M R Johnson

\section{Practical psychiatric epidemiology}

Martin Prince, Robert Stewart, Tamsin Ford, Matthew Hotopf, eds. Oxford: Oxford University Press, 2003, pp 401, £35.00. ISBN 0-19-851551-0

It is a pleasure for a reviewer to recommend a book wholeheartedly and this I am happy to do with the present volume. It lives up to its title of taking a practical view of the topic of psychiatric epidemiology and the editors have ensured a graded approach from basic principles and their application, into the more esoteric areas of interpretation of results and the consideration of more specialised and newly developing aspects of the subject.

This is not to say that it is a particularly easy book to read and the dilettante reader might well find it discouraging in its degree of detail. On the other hand, for the aspiring psychiatric epidemiologist or, just as importantly, for any researcher in psychiatry who wishes to make valid use of epidemiological methods, this book is required reading. As only one example of the latter, anyone planning to undertake a trial of a new psychotropic drug should carefully read the descriptions of potential bias in sample selection and category assignment.

I doubt if the average psychiatrist will want to buy a book on this rather specialised and research orientated subject but it should certainly be in every psychiatric library (and not just in the UK). It would be extraordinarily useful as a class text for a formal course in psychiatric epidemiology with a knowledgeable teacher acting as guide.

The editors have ensured a high quality of presentation throughout and, despite multiple authorship, there is relatively little repetition.

If I have a cavil it is not about the book but about the topic. In their introductory chapter the editors bemoan the lack of demonstrable validity of many modern research results obtained by using contemporary diagnostic and epidemiological methodologies (p 7). They also express a yearning for the discovery of genetic markers applicable to psychiatric illnesses (p 8). One can only say "Amen" to these comments because so often at present we find ourselves chasing shadows. However, I think there is increasing cause for optimism as neuropsychiatric research becomes increasingly finer grained.

As this book demonstrates, when the objective criteria emerge, there are tried and reliable methods standing ready to be applied.

A Munro

\section{Self-assessment colour review of clinical neurology and neurosurgery}

Neil Kitchen, Hadi Manji, Guy M McKhann II. London: Manon Publishing, 2003, pp 192, £16.95. ISBN 1-84076-011-7

Time was when none of us would have considered voluntarily using self-assessment as a form of learning. Exams were feared and to be avoided. This book shows that some progress has been made and modern learning methods may have some validity! It is a small slim book, which makes a welcome change. It is based on the idea of using self-assessment to aid and encourage learning. On the right hand pages are a series of questions with the 
answers on the succeeding left hand page to prevent easy cheating! The questions are randomly set, which avoids prejudice but does not allow someone to revise a particular topic very easily. Most of the questions are based on spot diagnoses, particularly of CT scans. The pictures and scans are very well reproduced and of much higher quality than in many textbooks. The discussion and explanations are generally well written and informative. It is up to date and has many questions on management. However, there are too many acronyms. Although there is a good glossary at the front, it is irritating for the novice to be repeatedly flicking back and forwards.

The introduction does not clearly state the target audience. It is too complicated for MRCP candidates in this country; and neurology registrars, to whom I showed it, found it too simple-while they might enjoy a quick glance on a train journey, I doubt whether they would buy it. Because the questions are based largely around CT scans or other illustrations, most are spot diagnoses rather than the complicated case histories of the NEJM CPC kind with an illuminating differential diagnosis; so there are few insights into the diagnostic process.

D E Bateman

\section{The asymmetrical brain}

Kenneth Hugdahl, Richard J Davidson, eds. Massachusetts: MIT Press 2003, pp 776, \$90.00. ISBN 0-262-08309-4

Clinicians probably most commonly encounter brain asymmetry in respect of language function. However, asymmetries, both anatomical and functional, exist at all levels of the nervous system. In this edited volume, Hughdahl and Davison present 21 varied chapters that range widely over current thinking about functional asymmetries and their correlates in brain anatomy. In presenting their field, the editors have chosen a mixed selection of chapters that range from basic physiological processes at the neuronal level through to major clinical disorders such as schizophrenia and depression. The major techniques used in the study of brain asymmetry, such as neuroanatomy, functional brain imaging, and transcranial magnetic stimulation are given their own descriptive chapters, but the bulk of the book is devoted to chapters that describe basic scientific studies of visual, auditory, and emotional laterality. With respect to clinical disorders, the major psychiatric disorders (schizophrenia and depression) are given their own chapters but the clinical neurologist may feel a little disappointed that only callosal agenesis and dyslexia are discussed in the section on neurological disorders. However, this is a minor criticism; the strength of the book is the breadth of research presented, and the description of how newer neuroscientific techniques are being brought to bear on longstanding questions of functional and anatomical asymmetry in the human brain.

\section{G Rees}

\section{Disorders of neuronal migration}

Peter G Barth, ed. Cambridge: Cambridge University Press 2003, pp 194, \$45.00 (hardback). ISBN 1-898-68331-X

This is an excellent, concise book that is written by the leaders in this rapidly growing subject. Peter Barth is to be congratulated on producing such a helpful and timely volume.

The book is written from the perspective of a clinician who wants to understand the relevance of the latest embryological, genetic, magnetic resonance, and experimental data on migration disorders and it is of particular relevance to paediatric neuroscience.

For example, the chapter on morphogenesis of the human cerebral cortex by Cainess, Takahashi, and Nowakawski, makes regular reference to important pathological mechanisms while describing normal development in a way that is most refreshing. The account of Lissencephaly by Dobyns and Leventer is masterly. There are also very nice summaries of the role of excitotoxic damage by Gressens, Barkovich, and Evrard, and of fetal disruption by Peter Barth.

It is not possible for a book of this size so well referenced to be clinically comprehensive and I think that that is reasonable recognising the imperative of providing good information on genetics, neuroradiology, and pathology. I think, therefore, that it is essential reading for those concerned with developmental and paediatric neuroscience.

B Neville

\section{Women with epilepsy: a handbook of health and treatment issues}

Martha J Morrell, Kerry Flynn, eds. Cambridge University Press, Cambridge, 2003, pp 290, $£ 55.00$. ISBN 0-521-6522-3.

This book was written in recognition of our increasing knowledge of the way in which epilepsy affects women specifically, and examines the issue from a biological, psychosocial, and treatment standpoint. Its aim was to provide information both for women with epilepsy and those around them, including their clinicians, families, and friends. It is a wide ranging book, covering such diverse areas as a historical perspective of the woman with epilepsy, the epidemiology, classification, and genetics of epilepsy, hormonal issues and sexual dysfunction in women with epilepsy, family planning, pregnancy and teratogenicity, legal and safety issues, and the psychosocial impact of epilepsy.

Inevitably in a book of this nature directed at a varied audience, there is a tension between clarifying the information sufficiently for lay readers while maintaining the interest of the clinician. Generally the authors succeed in this balance, but I felt that some of the chapters, particularly those addressing hormonal issues, remained rather technical for the lay reader. Other sections, such as those on the impact of epilepsy on relationships and psychiatric complications in epilepsy, were clearly written and provided useful information rarely available in standard textbooks, although the chapter on legal issues facing women with epilepsy was unfortunately of limited relevance to those outside the US.

The need for more information about the impact of epilepsy on women has been widely acknowledged in recent years, and this book will be of great interest not only to women with epilepsy and their families, but also to their doctors and epilepsy nurse specialists.

Y Hart

\section{Unipolar depression: a lifespan perspective}

Ian $M$ Goodyer, ed. Oxford: Oxford University Press 2003, pp 203, £24.95. ISBN 0-19851095-0

At first glance this is a very unimposing book, with a cover design that makes little impression and barely 200 pages for so important a subject. The prevalence of major depressive disorder is $0.4-2.5 \%$ in school aged children, $3-8 \%$ in older adolescents and adults, and up to $16 \%$ in later life. This clearly has a massive impact on all aspects of the community and on the large number of people who suffer depression.

Multi-author books often show a lack of balance between chapters, giving each topic the same level of importance, and often have duplication and overlap of subject matter. This book manages to avoid these problems, in large part because of the way it is structured in taking the reader through the various stages of life: infancy and pre-school years, the school aged child, adolescence, early adult life, mid life, and later life. This chronological, or maturational, perspective gives a much better appreciation of the intimate interactions of biological/genetic factors and experiences. Reading the book with this perspective is also in some ways a moving experience, as if watching and anticipating the development and progression of the illness in an individual.

The book takes us through the literature up to and including 2002, which is as good as can be expected for a book published last year. The review of the literature is thorough and gives a balanced perspective. This is in large part because the authors are experts in the field and manage to write in a clear and pragmatic way linking clinical management with up to date research.

It is a cliché to say you should not judge a book by its cover and this is a clear example of it. I am very happy to be able to keep this copy.

M Maier

\section{CORRECTION}

doi: 10.1136/jnnp.2004.015354corr l

In the short report by Pohlmann-Eden et al (J Neurol Neurosurg Psychiatry 2004;75: 898-900) the last author's name is spelt incorrectly, and should read I Blumcke. 